\title{
Blind Restoration of Motion Blurred Image by Applying a Non-iterative Algorithm
}

\author{
Shinichi Kuroyanagi, Ryota Maruo, Yukihiro Kubo and Sueo Sugimoto \\ Dept. of Elecrical and Electronic Engineering, Ritsumeikan University \\ 1-1-1 Noji-Higashi, Kusatsu City, Shiga 525-8577, Japan \\ E-mail:sugimoto@se.ritsumei.ac.jp
}

\begin{abstract}
In this paper, we present blind image restoration algorithms for motion blur caused by a motion between the imaging equipment and the object during the exposure time. We explain the motion degradation analysis and how to estimate parameters using Cepstral analysis, Radon transform and Hough transform.
\end{abstract}

\section{Introduction}

Motion blurred image is degraded image caused by the relative motion between the camera and the captured object. On the other hand, there are many kinds of degraded images. Especially we hit upon two kinds of images. The first one is linear motion blurred image, the second one is rotational motion blurred image. In our research, we focus on linear and rotational motion blurred images. We have already developed blind image deblurring algorithms for linear motion blurred images $[1,2]$ and for the rotational motion blurred images under the assumption of the known center of the rotation $[3,4,5]$. Then, we introduce the parameter estimation methods, cepstral analysis and Radon transform apply the estimation of the blur length and motion direction in the linear motion blurred images. Hough transform apply to estimate the center of rotation in rotational motion blurred images. To restore the blurred image, it needs to estimate parameters which show the properties of motion blurres. The blurred image is restored by using an inverse filter and the method of Lagurange multipliers. Experimental results show the feasibility and reliability of the method.

\section{Linear Motion Degradation Analysis}

A motion blurred image $g(x, y)$ can be modeled as an output of 2-D convolution process between true image $f(x, y)$ and point spread function which is function blurring the $h(x, y)$. In the noiseless case, the relationship between the observed image $g(x, y)$ and true image $f(x, y)$ is described as follows:

$g(x, y)=\int_{-\infty}^{\infty} \int_{-\infty}^{\infty} f\left(x^{\prime}, y^{\prime}\right) h\left(x-x^{\prime}, y-y^{\prime}\right) d x^{\prime} d y^{\prime}$

$$
=f(x, y) \otimes h(x, y)
$$

If velocity $(V)$ of the captured objects is assumed constant during the exposure time $T$ with an angle $\theta$ from the horizon, then the distortion is one dimensional. if $L=V T$, the PSF $h(x, y)$ of linear motion in spatial domain can be described as follows.

$$
\begin{aligned}
& h(x, y) \\
& = \begin{cases}\frac{1}{L} ; & 0 \leq|x| \leq L \cos \theta, \quad 0 \leq|y| \leq L \sin \theta \\
0 ; & \text { otherwise }\end{cases}
\end{aligned}
$$

The frequency response of $h(x, y)$ applying the Fourier transform is the $S I N C$ function. This is described as follows.

$$
\begin{aligned}
H\left(\omega_{x}, \omega_{y}\right) & =\mathcal{F}\{h(x, y)\} \\
& =\operatorname{sinc}\left\{\pi L\left(\omega_{x} \cos \theta+\omega_{y} \sin \theta\right)\right\}
\end{aligned}
$$

Suppose that an image $f(x, y)$ affects planar motion, and let $\left(x_{0}(t), y_{0}(t)\right)$ be the time-varying components of motion along $x$-axis and $y$-axis, respectively, and the image obtaining by this process is defined as $g(x, y)$. The total exposure at any points of the recording medium is obtained by integrating the instantaneous exposure over the time interval while the shutter of camera is open. Then let $t_{\text {exp }}$ be the duration of the exposure, then the blurred image in a general form can be defined as $[1,2]$

$$
g_{\operatorname{lin}}(x, y)=\frac{1}{t_{\exp }} \int_{0}^{t_{\exp }} f\left(x-x_{0}(t), y-y_{0}(t)\right) d t
$$

where $x_{0}\left(t_{\text {exp }}\right)=0, y_{0}\left(t_{\text {exp }}\right)=0$. Considering these process, input pixel positions $(x, y)$ move on the linear line during the exposure $t_{\text {exp }}$ in Fig. 1.

\section{Rotational Motion Degradation Analysis}

In the Cartesian system, rotational motion blurred image is constructed by the shift variant operation. Fig. 2 is the difference between shift variant and shift invariant in the Cartesian system. Before considering operation, we must consider that this method has the following two problems. First, for the given angle of rotation, the length of the rotational motion that affects 


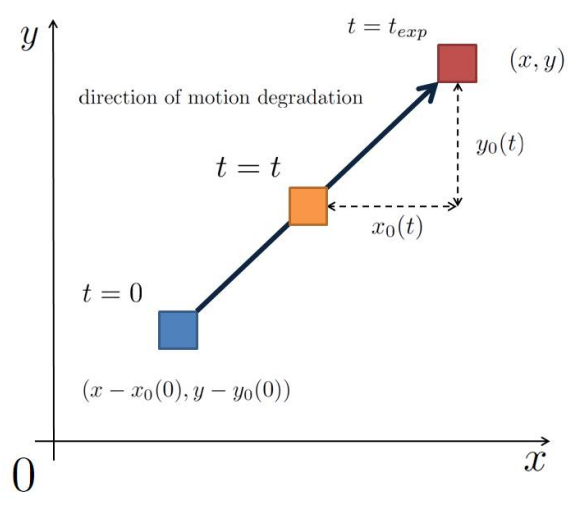

Fig 1: Concept of linear motion

the image pixels will be different for each rotational motion path. Since each rotational motion path has different radius measured from the center of rotation, values of the blur magnitude is influenced by the radius. Second, each image pixel will be blurred differently depending on its distance to the center of rotation. If the image pixels are further from the center of rotation, the image pixels will be more blurred. For these reasons, it is impossible to use the PSF as the linear filter and to use (4). Thus, it is necessary to treat the motion degradation on the rotational path as a problem of the linear motion degradation. With that in mind, we consider each input image pixel linear motioned on the rotational path.
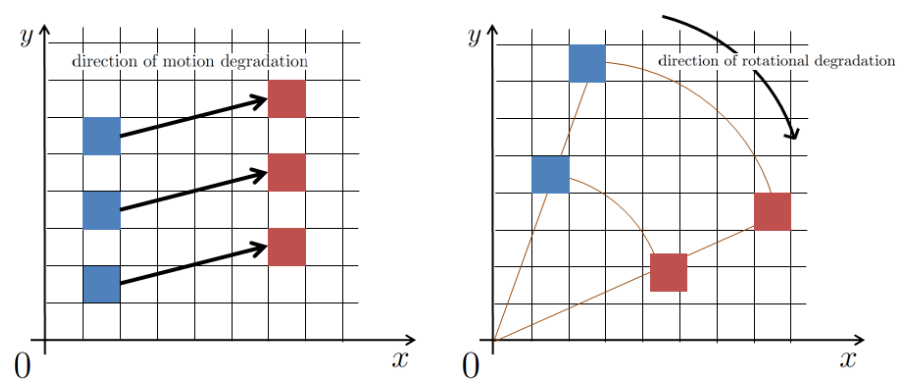

Fig 2: The difference of shift invariant and shift variant

\subsection{Motion Degradation in Cartesian Coordi- nate System}

Considering the rotational motion blur, the pixels which construct an input image move on the rotational path in Fig. 3. where $\left(x_{c}, y_{c}\right)$ means the position of the center of rotation. We introduce (4) in Fig. 3 to treat the operation on the rotational path.

Considering to introduce the parameters of radius $r$ and angular displacement $\gamma(0 \leq \gamma \leq R)$ in Fig. 3, construction of the pixel positions in the Cartesian coordinate system can be developed in Fig. 3. $\psi(t)$ which

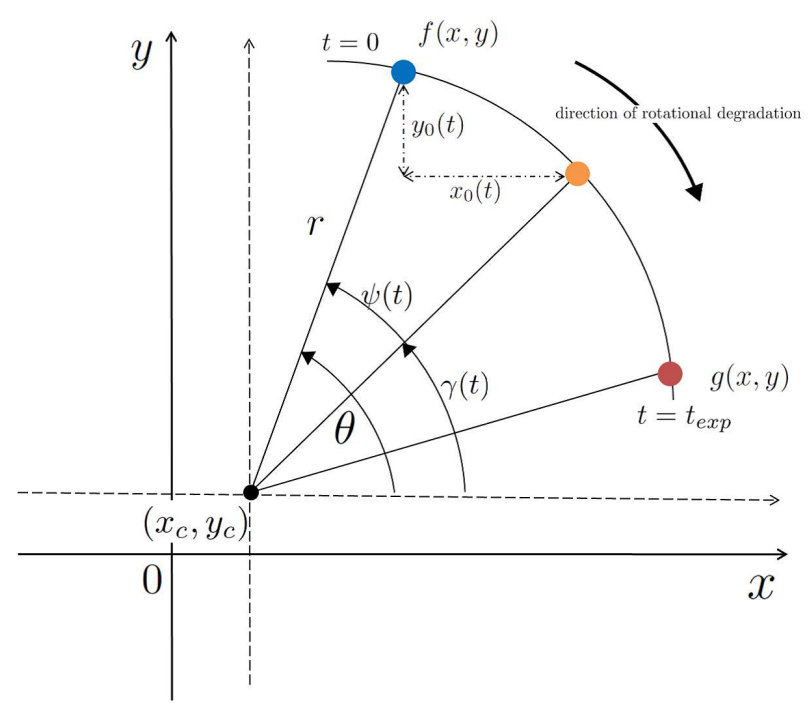

Fig 3: Construction of the pixel position in rotational motion degradation

means the angular displacement measured from the position of input image pixel at exposure time $t$ can be expressed as [3]

$$
\psi(t)=\omega t
$$

where $\omega$ is the constant angular velocity. In addition, we can write the following relation:

$$
\theta=\gamma(t)+\psi(t)
$$

To descrive the blurred image by the motion component along the rotational path $(\psi(t))$, we rewrite (6) as

$$
\psi(t)=\theta-\gamma(t)
$$

In general, in the Polar coordinate system, any Cartesian $(x, y)$ coordinate points can be expressed as[4]

$$
\begin{array}{r}
r=\sqrt{\left(x-x_{c}\right)^{2}+\left(y-y_{c}\right)^{2}} \\
\theta=\tan ^{-1}\left(\frac{y-y_{c}}{x-x_{c}}\right)
\end{array}
$$

By using (8) and (9), the radius $r$ and the angular displacement $\gamma(t)$ at exposure time $t\left(0 \leq t \leq t_{\text {exp }}\right)$ can also be expressed as

$$
\begin{array}{r}
r=\sqrt{\left(x-x_{0}(t)-x_{c}\right)^{2}+\left(y-y_{0}(t)-y_{c}\right)^{2}} \\
\gamma(t)=\tan ^{-1}\left(\frac{y-y_{0}(t)-y_{c}}{x-x_{0}(t)-x_{c}}\right)
\end{array}
$$

Considering (10) and (11), (4) which means the rotational motion blurred image treated linearly can be expressed as

$$
\begin{aligned}
& g_{\mathrm{rot}}(x, y)=\frac{1}{t_{\exp }} \int_{0}^{t_{\exp }} f\left(r \cos (\gamma(t))+x_{c},\right. \\
& \left.r \sin (\gamma(t))+y_{c}\right) d t
\end{aligned}
$$




\subsection{Image Description for Polar Coordinate System}

The circular motion in the Cartesian coordinate system is transformed into the line motion in the Polar coordinate system. For this purpose, the Polar coordinate transformation and interpolation of image pixels are required, where the bicubic interpolation method is applied in this paper. Therefore, considering Fig. 4, Cartesian input image pixels on the rotational path can be transformed into lines in the Polar coordinate system. For this reason, the discrete rotational motion blurred image in the polar coordinates $z(r, \theta)$ can be expressed as the summation of unblurred image in space polar coordinates $u(r, \gamma)$

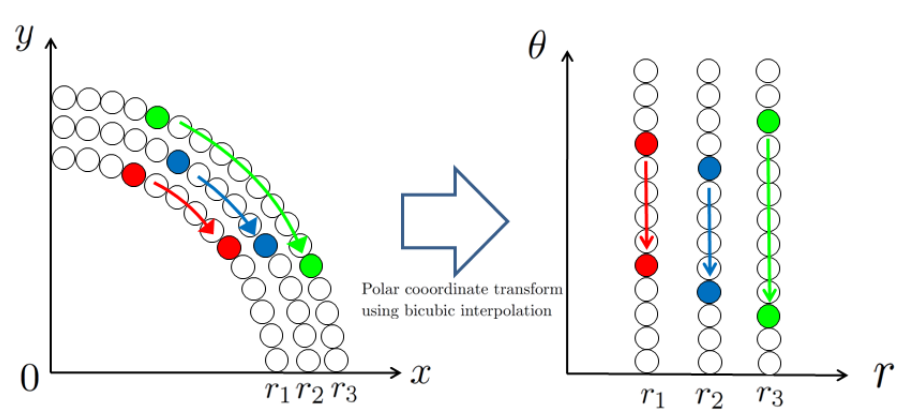

Fig 4: Polar coordinate transform

\subsection{Blurring Function Models \\ 3.3.1 LSF of the Rotational Motion Blur}

Although the in-plane rotational motion blurred image is spatially varying in the Cartesian coordinate system, it should be expressed as an output of the shift invariant system to alleviate the restoration. Such consideration can be achieved since the rotational motion blurred image is analogous with the linear motion blur along the rotational path [2]. Here, it is important to express the angular displacement as the arc length. The relation between them can be obtained by using the radius of the rotational path. By using the variable arc length $\tilde{\omega}=r \omega$ to replace $\omega, \tilde{\theta}=r \theta$ to replace $\theta$, (5) and (7) becomes

$$
\begin{array}{r}
\tilde{\psi}(t)=\tilde{\omega} t \\
\tilde{\psi}(t)=\tilde{\theta}-\tilde{\gamma}(t)
\end{array}
$$

Here, to operate the shift-invariant system in the blurring paths, it is necessary to express the blurred process by using parameters of the radius and the angle. In the Polar coordinate space, by using (13), (14), the blurring process for the constant angular velocity case referring (12) can expressed as [4]

$$
\begin{aligned}
\tilde{g}_{\mathrm{rot}}(r, \tilde{\theta}) & =\frac{1}{t_{\exp }} \int_{0}^{t_{\exp }} f(r, \tilde{\psi}(t)) d t \\
& =\frac{1}{t_{\exp }} \int_{0}^{t_{\exp }} f(r, \tilde{\omega} t) d t .
\end{aligned}
$$

From (13), considering the variable of arc length, we have

$$
\frac{d \tilde{\psi}}{d t}=\tilde{\omega}
$$

Here, it is important to transform $g$ to $g_{r}$, and $f$ to $f_{r}$ for adopting to use shift invariant operation in the Polar coordinate space. $g_{r}$ and $f_{r}$ mean an input image and a blurred image on each blurring path constructed by radius $r$. In addition, they are used in referring to the partial differentiation by radius $r$ to stress the angular displacement of the image pixels. Thus, by using $g_{r}$ and $f_{r}$, the shift invariant motion blur along the rotational motion path can be expressed in the general form as

$$
g_{r}(\tilde{\theta})=\int_{-\infty}^{\infty} f_{r}(\tilde{\psi}) h(\tilde{\theta}-\tilde{\psi}) d \tilde{\psi} .
$$

For each $r$, by using (17), (15) becomes

$$
\begin{aligned}
g_{r}(\tilde{\theta}) & =\frac{1}{t_{\text {exp }}} \int_{\tilde{\theta}-\tilde{\omega} t_{\text {exp }}}^{\tilde{\theta}} f_{r}(\tilde{\psi}) \frac{1}{\tilde{\omega}} d \tilde{\psi} \\
& =\int_{\tilde{\theta}-\tilde{\omega} t_{\text {exp }}}^{\tilde{\theta}} f_{r}(\tilde{\psi}) \frac{1}{\tilde{\omega} t_{\text {exp }}} d \tilde{\psi} \\
& \approx \frac{1}{L} \sum_{i=0}^{L-1} f_{r}\left(\tilde{\theta}-i \frac{\tilde{\omega} t_{\text {exp }}}{L-1}\right)
\end{aligned}
$$

Either (18) or (19) expresses the one dimensional degradation process. Under the shift invariant degradation process, the line spread function $(L S F)$ of the rotational motion blur along each rotational path can be obtained from (18) and (19). If $l$ is the index of pixels along the rotational motion path, we have

$$
l=\tilde{\psi}
$$

Therefore, the line spread function is given by

$$
h(l)= \begin{cases}\frac{1}{\tilde{\omega} t_{e x p}} & 0 \leq l \leq \tilde{\omega} t_{\text {exp }} \\ 0 \quad \text { otherwise }\end{cases}
$$

In (19), $L$ is the rectangle function given by

$$
L=\left[\frac{\Phi}{360} 2 \pi r\right]
$$

where $\Phi$ mean the given the rotation angle and the truncation operation, respectively. By using (22), $\tilde{\omega} t_{\text {exp }}$ in (21) can be expressed as the motion length along the rotational motion path. The length of this rectangle function is $L=\tilde{\omega} t_{\text {exp }}$. As the rotational motion blurred image is spatially invariant in the Polar coordinate form, it becomes similar to a linear motion blurred image on the polar coordinate.

\section{Parameter Estimation}

\subsection{Estimation of motion length}

In this paper, motion length estimation is done by applying cepstral analysis. Cepstral is the inverse Fourier 
transform of the logarithm spectrum of a signal. cepstral of motion blurred image $\tilde{g}(x, y)$ is

$$
\begin{aligned}
\tilde{g}(x, y) & =\mathcal{F}^{-1}\left\{\log \left|G\left(\omega_{x}, \omega_{y}\right)\right|\right\} \\
& =\tilde{h}(x, y)+\tilde{f}(x, y)
\end{aligned}
$$

As a result, cepstral of motion blurred image is added cepstral of true image and PSF. $\tilde{h}(x, y)$ has a large negative peak at a distance $L$ from the origin. therefore, the blur length is estimated by calculating the distance of the large negative peaks from origin.

\subsection{Estimation of Motion Direction}

In image processing, the Radon transform is the projection of the image intensity along a radial line oriented at a specific angle. parameters $\rho$ and $\theta$, Mathematically, it is written as

$\mathcal{R}(g, \rho, \theta)=\int_{-\infty}^{\infty} \int_{-\infty}^{\infty} g(x, y) \delta(x \cos \theta+y \sin \theta-\rho) d x d y$

The symbol $\mathcal{R}$, denoting the Radon transform operator. Eq. (24) can be expressed as

$\mathcal{R}(g, \rho, \theta)=\int_{-\infty}^{\infty} g(\rho \cos \theta-s \sin \theta, \rho \sin \theta+s \cos \theta) d s$

In Eq. (25), $\rho$ and $s$ can be calculated from $x, y$ and $\theta$ by using $\rho$ and the following equation, respectively

$$
\begin{aligned}
& \rho=x \cos \theta+y \sin \theta \\
& s=-x \sin \theta+y \cos \theta
\end{aligned}
$$

or

$$
\begin{aligned}
& x=\rho \cos \theta-s \sin \theta \\
& y=\rho \sin \theta+s \cos \theta
\end{aligned}
$$

The straight line in the image can be found by using the Radon transform. The straight line with $\left(\rho^{*}, \theta^{*}\right)$ can be written as

$$
g(x, y)=\delta\left(\rho^{*}-x \cos \theta^{*}-y \sin \theta^{*}\right)
$$

Therefore,

$$
\begin{array}{r}
\mathcal{R}(g, \rho, \theta)=\int_{-\infty}^{\infty} \delta\left\{\rho^{*}-(\rho \cos \theta-s \sin \theta) \cos \theta^{*}\right. \\
\left.-(\rho \sin \theta+s \cos \theta) \sin \theta^{*}\right\} d s \\
=\int_{-\infty}^{\infty} \delta\left\{\rho^{*}-\rho \cos \left(\theta-\theta^{*}\right)+s \sin \left(\theta-\theta^{*}\right)\right\} d s
\end{array}
$$

If $\theta \neq \theta^{*}$, Eq. (27) can be written as follows.

$$
\mathcal{R}(g, \rho, \theta)=\frac{1}{\left|\sin \left(\theta-\theta^{*}\right)\right|}
$$

if $\theta=\theta^{*}$, Eq. (27) can be written as follows.

$$
\begin{aligned}
\mathcal{R}(g, \rho, \theta) & =\int_{-\infty}^{\infty} \delta\left(\rho^{*}-\rho\right) d s \\
& = \begin{cases}0 & \rho^{*} \neq \rho \\
\int_{-\infty}^{\infty} \delta\left(\rho^{*}-\rho\right) d s & \rho^{*}=\rho\end{cases}
\end{aligned}
$$

Eq. (28) and (29) imply that the peak is formed when $\rho=\rho^{*}, \theta=\theta^{*}$. Therefore, from the peak value of the Radon transform, we can get the corresponding line parameter, namely $\rho$, and $\theta$. In other words, the Radon transform can be used to detect the line whose one of its parameters is $\rho$ and $\theta$.

\subsection{Center of Rotation Estimation}

For rotational motion blurred image, it is important to estimate the center of rotation. Degradation image filters using Laplacian filter mask and change this image for binarize image. This binarized image is $g^{\prime}(x, y)$. It is applied Hough transform and extract the center of rotation. $\left(x_{c}, y_{c}\right)$ is the center of rotation. $r$ is radius. $(x, y)$ is point that has pixel value on $g^{\prime}(x, y)$. By using Laplacian filter mask, the detected arc is applied a circle including $\left(x_{c}, y_{c}\right)$ and $r$. Then Hough transform is defined as follows

$$
\left(x-x_{c}\right)^{2}+\left(y-y_{c}\right)^{2}=r^{2}
$$

We can therefore construct a 3D accumulator $N\left(x_{c}, y_{c}, r\right)$. For each edge point vary $\left(x_{c}, y_{c}\right)$, compute the corresponding $r$ using the circle equation, and the update $\mathrm{A}$. In the end, search $\mathrm{N}$ for peaks. If we know the gradient direction of each point, we may use a different parameteric representation for circle to take advantage of the gradient direction

$$
\begin{aligned}
& x=r \cos \theta+x_{c} \\
& y=r \sin \theta+y_{c}
\end{aligned}
$$

where $\theta$ represents the gradient direction of point $(x, y)$. We can then reduce search from $2 \mathrm{D}$ to $1 \mathrm{D}$. We can vary $r$ and then use the above equation to compute $x_{c}$ and $y_{c}$, then updating the corresponding accumulator accordingly. For the parametric cirle equation, we have the tangent of the circle at $(x, y)$ is

$$
\frac{d x}{d y}=-\tan \theta
$$

From circle equation $\left(x-x_{c}\right)^{2}+\left(y-y_{c}\right)^{2}=r^{2}$, we have

$$
\frac{d x}{d y}=-\frac{y-y_{c}}{x-x_{c}} .
$$

Equating the above two equations yields

$$
\frac{y-y_{c}}{x-x_{c}}=\tan \theta
$$

where $\theta$ represents the gradient direction at $(x, y)$. It transforms $(x, y)$ on the Cartesian coordinates into 3D parameters space $N\left(x_{c}, y_{c}, r\right)$, so it corresponds to a single plane. If there are many points on Cartesian coordinates, many surfaces can be obtained on 3D parameters space. Because a circle is shown the three parameters $\left(x_{c}, y_{c}\right)$ and $r$, it obtains a circle on a point in $3 \mathrm{D}$ parameters space $\left(x_{c}, y_{c}, r\right)$. Thorefore we vote on all of circles in the degradation image filters using 
Laplacian filter mask to 3D parameters space, and we can obtain the parameter of the circle by surveying the result of voting. Examine the count of the accumulator cells on 3D parameters space for high pixel concentrations. The highest point is requiring point $N\left(\hat{x}_{c}, \hat{y}_{c}, \hat{r}\right)$. So, we determine the center of rotation which is estimated as $\left(\hat{x}_{c}, \hat{y}_{c}\right)$.

\section{Image Restoration}

Let the motion blurred image, PSF and true image are denoted as $\mathbf{g} \in R^{K \times 1}, \mathbf{H} \in R^{K \times K}$, and $\mathbf{f} \in R^{K \times 1}$ respectively, then degraded image $\mathbf{g}$ is expressed by

$$
\mathrm{g}=\mathbf{H f} .
$$

Here, $\mathbf{H} \in R^{K \times K}$ and $\mathbf{f} \in R^{K \times 1}$ change to $\breve{\mathbf{H}} \in R^{K \times J}$ and $\breve{\mathbf{f}} \in R^{J \times 1}$ Since $\breve{\mathbf{H}}$ is not a square matrix and the size of $\breve{\mathbf{f}}$ is not similar to that of $\mathbf{g}$, matrix inversion can not be used to obtain $\breve{\mathbf{f}}$ from $\mathbf{g}$. To solve this problem, a regularization-based method is employed. the solution of the linear system $\breve{\mathbf{H}} \breve{\mathbf{f}}=\mathbf{g}$ is defined as $\hat{\mathbf{f}}$ whose components have the minimum distance to the observed data $\mathbf{g}$. Here, $\hat{\mathbf{f}}$ is a vector of the first $K$ elements of $\breve{\mathbf{f}}$ and is obtained by using

$$
\hat{\mathbf{f}} \equiv \mathbf{P} \breve{f}
$$

where $\mathbf{P}$ is an $K \times J$ matrix which projects the vector $\breve{\mathbf{f}}$ on the support of $\mathbf{g}$ Eq. (37) can be written as

$$
\left[\begin{array}{c}
\hat{\mathrm{f}}_{1} \\
\vdots \\
\vdots \\
\hat{\mathrm{f}}_{\mathrm{K}}
\end{array}\right] \equiv\left[\begin{array}{ccccccc}
1 & 0 & \cdots & \cdots & \cdots & \cdots & 0 \\
0 & 1 & \ddots & \ddots & \ddots & \ddots & \vdots \\
\vdots & \ddots & \ddots & \ddots & \ddots & \ddots & \vdots \\
0 & \cdots & 0 & 1 & 0 & \cdots & 0
\end{array}\right]\left[\begin{array}{c}
\breve{\mathrm{f}}_{1} \\
\vdots \\
\vdots \\
\vdots \\
\breve{\mathrm{f}}_{\mathrm{J}}
\end{array}\right]
$$

The problem of obtaining $\breve{\mathbf{f}}$ from $\mathbf{g}$ is a regularized least square problem defined as

$$
\begin{array}{r}
\underset{\breve{\mathbf{f}}}{\operatorname{minimize}} V(\breve{\mathbf{f}})=\underset{\breve{\mathbf{f}}}{\operatorname{minimize}}\|\mathbf{g}-\mathbf{P} \breve{\mathbf{f}}\|^{2} \\
\text { subject to }\|\mathbf{g}-\breve{\mathbf{H}} \breve{\mathbf{f}}\|^{2}=0
\end{array}
$$

This problem can be solved by using the Lagrange multipliers method. Here, we have a new function

$$
R(\breve{\mathbf{f}})=\|\mathbf{g}-\mathbf{P} \breve{\mathbf{f}}\|^{2}+\lambda\|\mathbf{g}-\breve{\mathbf{H}} \breve{\mathbf{f}}\|^{2}
$$

where $\lambda$ is the regularization parameter that controls the solution's degree of smoothness. The aforesaid formula has an analytical solution given by

$$
\breve{\mathbf{f}}=\underset{\breve{\mathbf{f}}}{\operatorname{argmin}} R(\breve{\mathbf{f}})
$$

Eq. (40) is realized by

$$
\frac{\partial R(\breve{\mathbf{f}})}{\partial \breve{\mathbf{f}}}=0
$$

Finally, we get

$$
\breve{\mathbf{f}}=\left[\lambda \breve{\mathbf{H}}^{\mathrm{T}} \breve{\mathbf{H}}+\mathbf{P}^{\mathrm{T}} \mathbf{P}\right]^{-1}[\lambda \breve{\mathbf{H}}+\mathbf{P}]^{\mathrm{T}} \mathbf{g}
$$

In Eq. (42), number of elements in $\breve{\mathrm{f}}$ is $J$ where $J=$ $K+1-L$. Therefore, the restored image pixels is the last $K$ elements of $\breve{\mathbf{f}}$ in Eq. (42).

$$
\check{\mathbf{f}}=\left[\begin{array}{llll}
\breve{\mathrm{f}}_{\mathrm{L}} & \cdots & \cdots & \breve{\mathrm{f}}_{\mathrm{J}}
\end{array}\right]^{T}
$$

\section{Simulation}

Our experiments are done by using MATLAB 2007a. The original image is shown in Fig. 5. Original image size is $256 \times 256$ pixels.

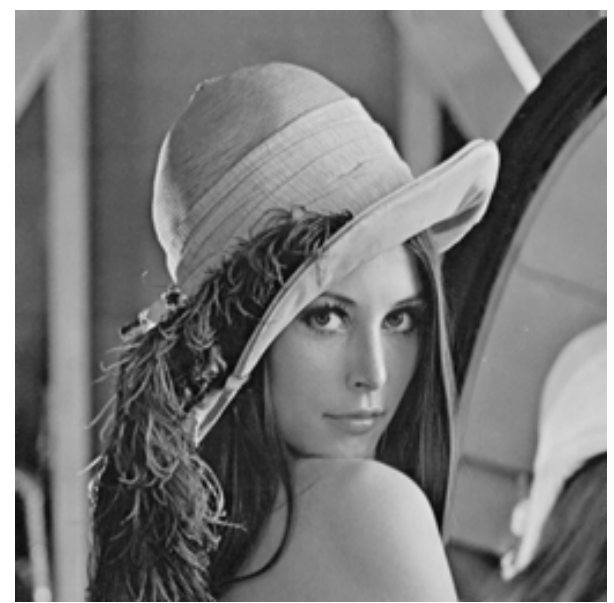

Fig 5: Original image

First, we show the result of blind restoration of linear motion blurred image. In our work, we use Cepstral analysis and Radon transform for estimating the motion length and direction. Table. 1 shows the estimated results using our methods. SNR is signal to noise ratio, shows the restoration degree between original and restored image.

Next, we show the results of image deconvolution. Lin-

Table 1: Estimation of motion length and direction

\begin{tabular}{|c|c|c|c|c|}
$\begin{array}{c}\text { True value } \\
\text { Motion length }\end{array}$ & $\begin{array}{c}\text { True value } \\
\text { Motion direction }\end{array}$ & $\begin{array}{c}\text { Estimated value } \\
\text { Motion length }\end{array}$ & $\begin{array}{c}\text { Estimated value } \\
\text { Motion direction }\end{array}$ & SNR \\
\hline 20 & 3 & 21 & 5 & 20.417 \\
\hline 20 & 33 & 20 & 33 & 20.409 \\
\hline 28 & 32 & 27 & 31 & 20.307 \\
\hline 30 & 11 & 30 & 11 & 20.171 \\
\hline 35 & 10 & 35 & 10 & 20.075 \\
\hline 40 & 20 & 40 & 19 & 19.877 \\
\hline
\end{tabular}

ear motion blurred image $(L=20$ pixels, $\theta=33$ degrees $)$ is shown in Fig. 6, and Fig. 7 is the restorated image 


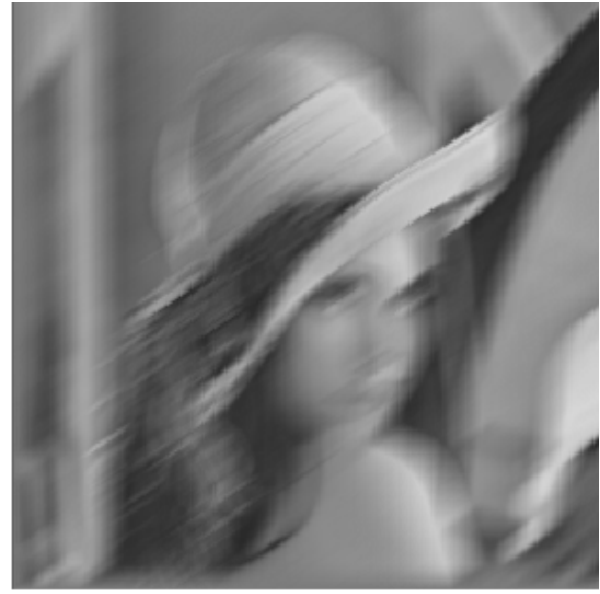

Fig 6: Linear motion blurred image

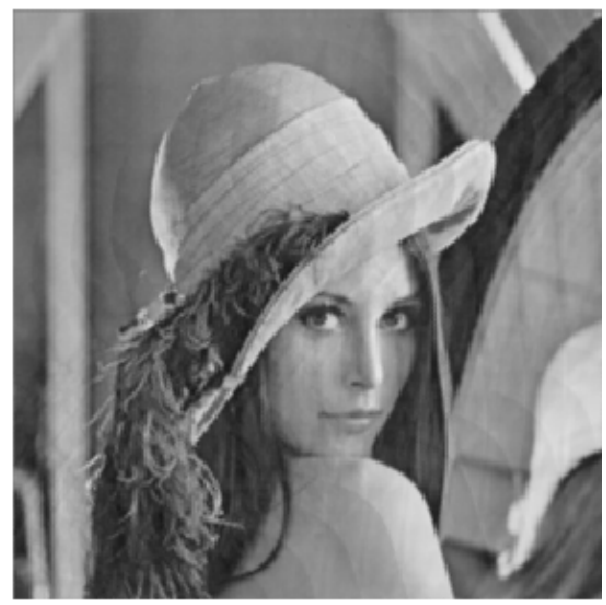

Fig 7: Restored image of motion blurred

using estimation parameters.

Next, we show the result of blind restoration of rotational motion blurred image. We use the Hough transformation for estimating the center of rotation $\left(\hat{x}_{c}, \hat{y}_{c}\right)$. And we use the Cepstral analysis for estimating motion length $\hat{L}$. Table. 2 shows the estimation results using our methods.

Table 2: Estimation Result

\begin{tabular}{|c|c|c|c|c|}
\hline $\begin{array}{c}\text { True value } \\
\text { Motion length }\end{array}$ & $\begin{array}{c}\text { True value } \\
\text { Center of rotation }\end{array}$ & $\begin{array}{c}\text { Estimation } \\
\text { Motion length }\end{array}$ & $\begin{array}{c}\text { Estimation } \\
\text { Center of rotation }\end{array}$ & SNR \\
\hline 3 & $(10.20)$ & 3 & $(11.24)$ & 4.10 \\
\hline 3 & $(30.30)$ & 3 & $(11.30)$ & 3.92 \\
\hline 5 & $(10.20)$ & 5 & $(13.25)$ & 2.95 \\
\hline 5 & $(30.30)$ & 5 & $(28.26)$ & 2.01 \\
\hline 10 & $(10.20)$ & 10 & $(28.26)$ & 1.51 \\
\hline 10 & $(30.30)$ & 10 & $(26.31)$ & 0.81 \\
\hline
\end{tabular}

Fig. 8 - 10 is the results of image deconvolution. The original image is transformed into the polar coordinated image where bicubic interpolation is applied. Then the rotational blurred image is obtained by transforming into the Cartesian image after summation of $L$ pixels along the $\theta$ direction in the polar coordinate, which is shown in Fig. 8, where the center of the rotation is assumed as $\left(x_{c}, y_{c}\right)=(10,20)$, and $L=3$. Further, we obtain the binary image in Fig. 8 by applying the Laplacian operator with 4 neighborhood for edge detection from Fig. 9. The Hough transformation is applied for obtaining the arcs of the circle so that we can estimate the center of rotation. In Fig. 9, red and green points show the estimated value $(11,24)$, the true value respectively. The estimated value is the result of voting, and it is the highest point. Table. 2 shows the estimated results of the center of rotation and the motion blurred length and SNR. We could not obtain the satisfactory results yet. Especially, the bigger motion length is, the more SNR does not have the ideal value. The restored image using the estimated value of $\left(\hat{x}_{c}, \hat{y}_{c}\right)$ and $\hat{L}$ is shown in Fig. 10.

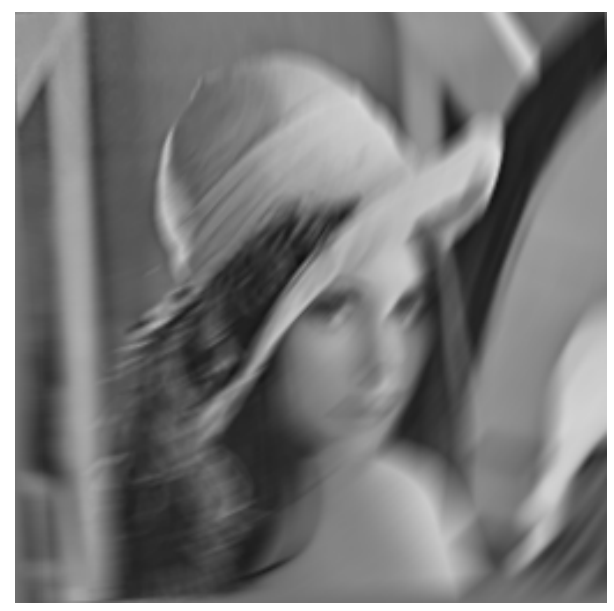

Fig 8: Rotaional blurred image 


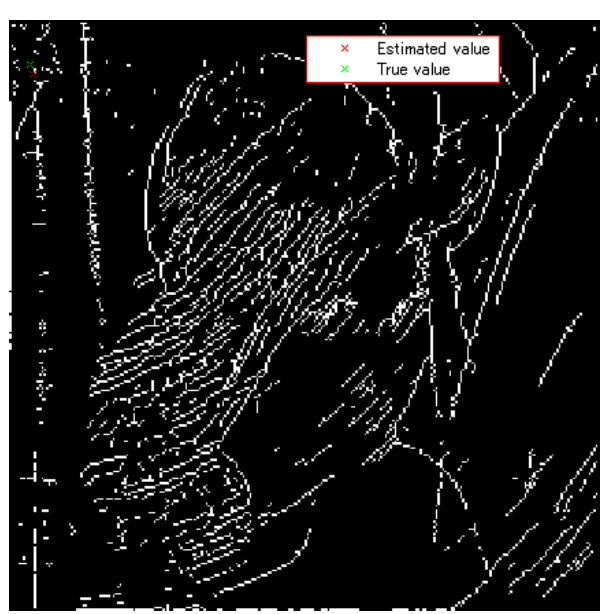

Fig 9: Rotational image filter Laplacian mask

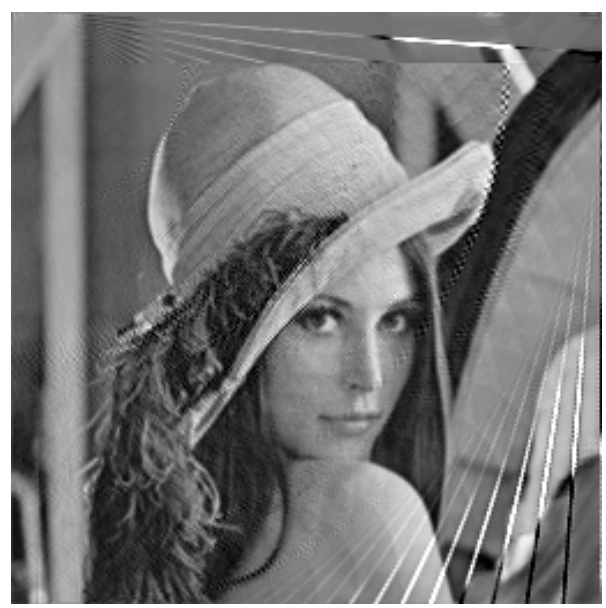

Fig 10: Restored image of rotational motion blurred

\section{Conclusions}

In this paper, we have presented an estimation method of the motion length, motion direction and the coordinate of the center of rotation. In linear motion, we can estimate the parameters of motion length and motion direction based on Cepstral analysis and the Radon transform, and we can restore the linear motion blurred image through the experimental results. In rotational motion, however, we could not obtain satisfactory restored images. These poor results may come from the errors due to many times of the transformation between the Polar and Cartesian coordinate systems. Also if we can obtain more accurate estimated values of the center of rotation, naturally we can obtain better restored images. Therefore we will develop the methods of detecting the arcs of circles more accurately in the near future.

\section{references}

[1] Wikky Fawwaz Al Maki, T. Kitagawa, T. Hori and Sueo Sugimoto: Shift Invariant Degradation Analysis and Restoration Method for Photograph Degraded by Rectilinear Smear, Proc. of the 40th ISCIE Intl Symp on Stochastic Systems Theory and Its Applications, pp. 166-170, Kyoto, Nov. 2008.

[2] N. Yokota, T. Hori, Wikky Fawwaz Al Maki and S. Sugimoto: Blur Estimation for Linear Motion Blurred Image: Proc. of the 42nd ISCIE Intl Symp on Stochastic Systems Theory and Its Applications, pp. 271-276, Okayama, Nov. 2010.

[3] Wikky Fawwaz Al Maki and Sueo Sugimoto: Removing Shift Variant Blur without Geometric Coordinate Transformation, SICE Journal of Measurement, and System Integration, Vol. 3, No. 2, pp. 67-74, March 2010.

[4] T. Morimoto, N. Isoe, Y. Ohizumi, W. Fawwaz Al Maki, and S. Sugimoto: Restoration of Rotational Motion Blurred Images using the Inverse Filters, Proc. of the 42nd ISCIE Intl Symp on Stochastic Systems Theory and Its Applications, pp. 264-270, Okayama, Nov. 2010.

[5] N. Isoe, R. Maruo, and S. Sugimoto: Blind Deconvolution of Rotational Motion Blurred Image Proc. of the 43rd ISCIE Int Symp on Stochastic Systems Theory and Its Applications, pp. 185-191, Shiga, Oct. 2011. 